\title{
Focused examination of cerebral blood flow in peri-resuscitation: a new advanced life support compliant concept
}

\author{
Lawrence A. Melniker
}

Received: 20 October 2010/Accepted: 2 November 2010/Published online: 3 December 2010

(C) Springer-Verlag 2010

\section{Dear Editor,}

I commend Haas et al. [1] for their report on the assessment of cerebral blood flow (CBF) with duplex sonography of the extracranial internal carotid artery (ICA) in the peri-resuscitation setting. The ALS-conforming addition to the FEEL exam seems simple, rapid, and cost-effective. The adequacy of CPR during resuscitation is a critical unknown; this new technique may shed needed light on this most critical situation. This report and case series generates important hypotheses that beg immediate investigation.

In paragraph 3 of the "Introduction", the authors bring up a vitally important point regarding pulseless electrical activity (PEA), but misstate its definition and, therefore, understate the significance of the issue. They define PEA "as 'clinical absence of cardiac output despite electrical activity' and essentially an echocardiographic diagnosis...equivalent to electromechanical dissociation [EMD]." For all practical purposes, PEA is simply the absence of pulses despite the presence of electrical activity and is a clinical, not an echocardiographic, diagnosis. Alternatively, EMD is truly the absence of cardiac mechanical activity (and, by extension, output) despite electrical activity and must be diagnosed manometrically or by echo. They are very different entities [2-5].

The EMD was abandoned more than 10 years ago precisely because of the presumption that clinicians were

\section{A. Melniker ( $\square)$}

Department of Emergency Medicine,

New York Methodist Hospital, 506 Sixth Street,

Brooklyn, NY 11215-9008, USA

e-mail: lam9004@nyp.org unable to assess cardiac activity during resuscitations. Since that time, the world has been plagued with the waste-basket term PEA, which encompasses both pulseless patients with EMD (who are often unresuscitatable) and those with cardiac activity and no palpable pulses (who are much more likely to achieve ROSC). With the increasing prevalence of clinical sonography in the periresuscitation setting allowing immediate assessment of cardiac activity, we can return to the utilitarian EMD and drop PEA into a linguistic dust bin, where it belongs.

Of critical importance, with the addition of CBF assessment via ICA duplex scanning, we may need to revisit the categorization of our pulseless patients. Perhaps there are three rather than just two critical patterns to identify: pulseless with EMD, pulseless with cardiac activity but inadequate $\mathrm{CBF}$, and pulseless with cardiac activity and adequate CBF. This may allow us to maximize the efficiency of our resuscitation efforts in new and important ways [6].

Conflict of interest None.

\section{Reference}

1. Haas M, Allendörfer J, Walcher F et al (2010) Focused examination of cerebral blood flow in peri-resuscitation: a new advanced life support compliant concept-an extension of the focused echocardiography evaluation in life support examination. Crit Ultrasound J 2:1-12

2. http://emedicine.medscape.com/article/161080-overview. Accessed 15 Oct 2010

3. Engdahl J, Bång A, Lindqvist J, Herlitz J (2001) Factors affecting short- and long-term prognosis among 1069 patients with out-ofhospital cardiac arrest pulseless electrical activity. Resuscitation 51(1):17-25 
4. http://medical.yourdictionary.com/electromechanical-dissociation. Accessed 15 Oct 2010

5. Ewy GA (1984) Defining electromechanical dissociation. Ann Emerg Med 13(9 Pt 2):830-832
6. SuYun Q, YunJuan L, Lei W, HeHua Y (2007) A clinical study on monitoring cerebral blood flow of children with cardiopulmonary resuscitation: P-085. Pediatr Int 49(5):755 